\title{
Imaging and Analysis of Void-Defects in Solder Joints Formed in Reduced Gravity Using High-Resolution Computed Tomography
}

\author{
John W. Easton ${ }^{1}$ \\ National Center for Space Exploration Research, Cleveland, OH, 44135 \\ Peter M. Struk ${ }^{2}$ \\ NASA Glenn Research Center, Cleveland, OH, 44135 \\ and \\ Anthony Rotella ${ }^{3}$ \\ Case Western Reserve University, Cleveland, $\mathrm{OH}, 44106$
}

\begin{abstract}
As a part of efforts to develop an electronics repair capability for long duration space missions, techniques and materials for soldering components on a circuit board in reduced gravity must be developed. This paper presents results from testing solder joint formation in low gravity on a NASA Reduced Gravity Research Aircraft. The results presented include joints formed using eutectic tin-lead solder and one of the following fluxes: (1) a no-clean flux core, (2) a rosin flux core, and (3) a solid solder wire with external liquid no-clean flux. The solder joints are analyzed with a computed tomography (CT) technique which imaged the interior of the entire solder joint. This replaced an earlier technique that required the solder joint to be destructively ground down revealing a single plane which was subsequently analyzed. The CT analysis technique is described and results presented with implications for future testing as well as implications for the overall electronics repair effort discussed.
\end{abstract}

\section{Introduction}

Tn recent years, NASA has set a series of goals for a continued, expanding presence in space. This includes 1 completion of the International Space Station (ISS), returning to and beginning a long duration mission to the moon, and a manned mission to Mars. These expanding missions, with longer distances and time away from Earth and ground-based material support, will require expanded self sufficiency by the crew of these missions. Launching spare or replacement parts, consumables, and new equipment on demand from Earth will be difficult for both lunar and Martian missions. Recent events on the ISS ${ }^{1,2}$ show that such support is difficult even for missions in low Earth orbit, and that unexpected problems arise even with highly planned and tested activities. One area where crew members can increase self sufficiency is in performing electronics repairs.

Given appropriate tools, training, and guidance from ground support teams, crew-performed electronics repairs can help increase the probability of mission success in a number of ways. First, it provides a recovery mechanism for unexpected failures by allowing crew members to repair failed components without sending faulty units to Earth and waiting for replacements. Having appropriate tools for electronics repairs decreases the risks involved in performing repairs with improvised tools and techniques, increases the reliability of repairs, and decreases the down time of faulty systems. Providing a repair capability will also reduce the mass and volume required for spare parts. While a repair capability will not eliminate the need for spare electronics units, it will reduce the number needed, allowing crew members to replace a faulty unit with a spare and then repair the faulty unit, which then becomes a spare itself. The reduction in mass and volume of spare parts then allows for the storage of other materiel or a

\footnotetext{
${ }^{1}$ Associate Staff Scientist, 21000 Brookpark Rd., Mail Stop 110-3, AIAA Member

${ }^{2}$ Aerospace Engineer, 21000 Brookpark Rd., Mail Stop 110-3, AIAA Member

${ }^{3}$ Student Researcher, 10900 Euclid Avenue
} 
reduction in the total vehicle mass. The Component Level Electronics Assembly Repair (CLEAR) project at NASA Glenn Research Center, Cleveland, $\mathrm{OH}$, is exploring and developing tools and processes to test, diagnose, and repair electronics at the lowest possible level, providing the advantages of electronics repair to future long duration missions $^{3,4,5}$.

A key aspect of performing electronics repairs in space is the ability, and consequences, of soldering in the selfcontained, reduced gravity environment of a space vehicle or habitat. Previous work has examined the effect of reduced gravity on the formation of solder joints ${ }^{6,7}$. This work studied the formation of solder joints in normal and reduced gravity, using $60 \%$ tin, $40 \%$ lead solders using rosin fluxes, both as a core material within the solder wire and as an external liquid used with a solid solder wire. In general, this work found that, using standard soldering techniques, solder joints produced in reduced gravity formed more internal voids than similar solder joints produced in normal gravity. Minimizing the formation of internal voids in solder joints is important because the voids can adversely affect the electrical conductivity, heat transfer, and mechanical strength of the solder joint. The authors hypothesize that gas bubbles within the molten solder joint cannot escape in reduced gravity due to reduced buoyant forces compared to normal gravity, thus increasing the porosity of the joint formed in reduced gravity. Further testing showed that solder joint voids can be reduced by using solid core solder wire with external liquid flux, and by increasing the amount of heat applied to the solder joint by keeping the soldering iron in contact with the joint for a longer time.

Recent tests conducted in the NASA Reduced Gravity Research Aircraft ${ }^{8}$ expand on this previous work by focusing on the use of eutectic solders and no-clean fluxes, both external liquid flux and flux-cored solder wire. The components of eutectic alloys solidify at the same temperature from the molten state; the authors hypothesize that this behavior could help in reducing the amount of voiding found in solder joints produced in low gravity. Eutectic alloys do not exhibit a plastic state, where one component begins to solidify while the other remains liquid when the temperature reduces from the melting point. This behavior may reduce the amount of voids formed within a solder joint by keeping the joint molten for a longer time than other tin-lead solder alloys. No-clean fluxes are expected to be important for use in electronics repairs during space missions, as these fluxes require little or no cleaning after use, reducing the requirements for water or isopropyl alcohol and decreasing containment concerns arising when using liquids in a reduced gravity environment.

The current work also uses a different analysis technique than those used in the work described above. In this case, computed tomography (CT) scans provided a sequence of images along the length of the entire solder joint, rather than a single cross section at one plane in the joint. This reduced the number of required samples for statistical analysis, and provides a more complete picture of solder joint formation in reduced gravity. The use of this analysis technique also required the development of an image analysis process to extract the relevant data from the CT images.

This paper will discuss the results of three sets of soldering tests in reduced gravity using eutectic solder; first with no-clean external liquid flux and solid solder wire; second using solder wire with a core of no-clean flux; third using solder wire with a core of rosin flux. All joints are analyzed using nondestructive CT analysis. The paper will first focus on the experiment setup and operation aboard the NASA Reduced Gravity Aircraft. It will also briefly describe the CT scanning process, and image analysis performed once the CT analysis was complete. Following this is a discussion of the various types of solder and flux tested, both in reduced gravity as well as in normal gravity for comparison. The solder and flux combinations will be compared with the results of this study as well as the previous studies already mentioned. Finally, the implications of these results will be discussed, as well as recommendations for use in reduced gravity as well as future work.

\section{Experiment Description and Data Analysis}

As already mentioned, this experiment focuses on determining the formation of solder void defects within solder joints formed in reduced gravity. The NASA Reduced Gravity Aircraft ${ }^{8}$ provided a facility for operating in reduced gravity. The modified C-9 aircraft flew a series of parabolic trajectories, allowing for 20-30 seconds of operation at gravity levels of $0.050 \mathrm{~g}$ or less. The experiment rig shown in Figure 1, bolted to the aircraft floor, provides a platform for forming solder joints, video recording the joint formation process, recording three-axis acceleration measurements on the rig, and providing power for lighting, the soldering tool, and data collection equipment. A glovebox section of the rig contained a soldering iron (Weller model TCP12), circuit board holder, and backlights. The glovebox itself was manufactured with Lexan windows for the user to see the operation, and glove ports for user access to the tools, circuit board, and other materials. A video camera with $105 \mathrm{~mm}$ lens recorded the solder process with an edge view of the circuit card, as shown in Fig. 2. An accelerometer, a Crossbow model CXL01LF3$\mathrm{R}$, output acceleration data which was recorded using a National Instruments CB-50LP data acquisition board with a 
National Instruments DAQCard 700 interface card and laptop computer. Acceleration data was recorded in all three axes at $100 \mathrm{~Hz}$, though only the vertical axis data was analyzed, as this was the direction of the g-vector. The data acquisition system also toggled a video overlay generator on or off, allowing for correlation between the video and acceleration data. This is the same experiment rig used in previous studies ${ }^{6,7}$, though the accelerometer and data acquisition systems used are new for this series of experiments.

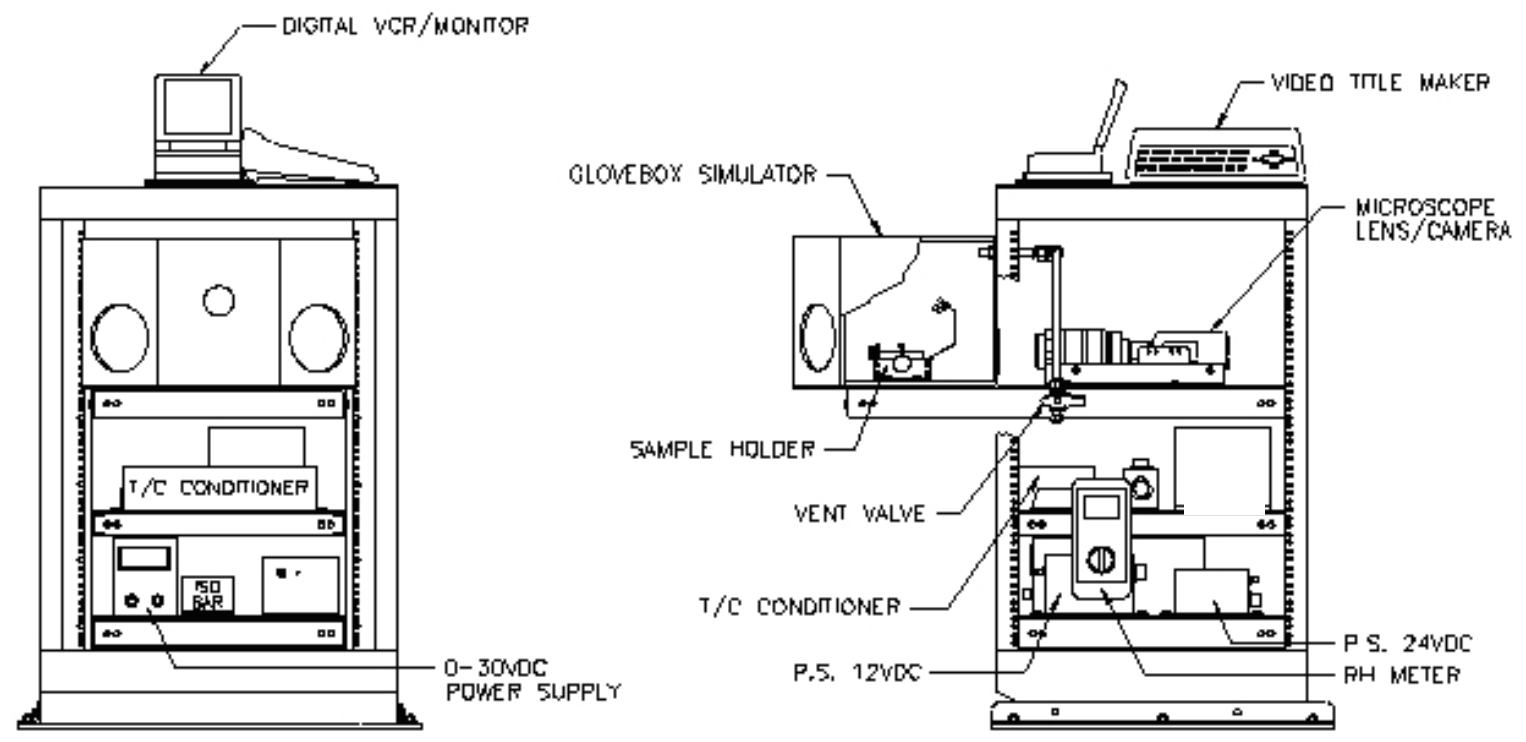

Figure 1. Schematic of aircraft test rig. Soldering operations are performed in the glovebox, with the rack contains support equipment.

The circuit boards used as test samples for these experiments allowed for simple mounting and alignment in the video camera field of view and minimized problems handling parts during aircraft operations. The circuit cards are two layer boards with plated through hole lands. Quarter watt resistors were attached to the circuit boards with an adhesive prior to use, with the resistor lead formed and inserted through a plated through hole. The experiment studied varying combinations of flux and solder, shown in Table 1 . These solders were all eutectic alloys of tin and lead (63\% tin, 37\% lead by weight). One solder wire had a core consisting of a noclean flux, a second wire type had a core of rosin flux, and the third solder was solid, with an external liquid no-clean flux applied prior to soldering. All solder wires had an outer diameter of 0.031 inches.

The experiment process proceeded as

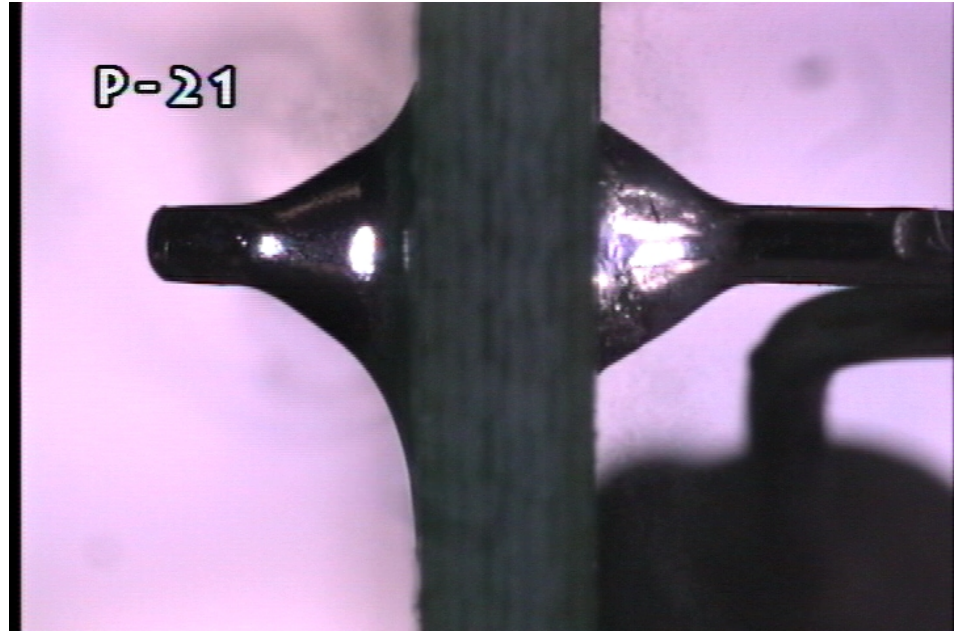

Figure 2. An image of a solder joint formed in reduced gravity, taken during operations on the research aircraft. The text in the upper left corner changes when acceleration data is recording or stopped recording. The image also shows the joint fillets, circuit board, and lead.

follows. Prior to the beginning of the reduced gravity portion of a parabola, the operator applied a droplet of external liquid flux on the joint area to be worked on, if the test matrix called for external flux. As gravity reduced, a coworker toggled a computer trigger to begin recording acceleration data and add an overlay character on the video stream. The operator waited for the gravity level to fall below $0.05 \mathrm{~g}$, then began soldering work. Using a "mental count," the operator heated the joint area with the soldering iron for three seconds. He then added solder to first form a heat bridge between the soldering iron, circuit board land and component leg, then continued adding solder to form 
the joint, and finally removed the solder wire. The operator kept the soldering iron on the joint for an additional three seconds and then removed the soldering iron. The timing pattern is that recommended by previous work ${ }^{6,7}$. At the end of the reduced gravity period, the assistant again toggled a computer trigger stopping acceleration data recording and removing the video stream overlay character. For comparison, four solder joints using the same solder and flux combinations as well as equipment used in the flight were formed in normal gravity, using the same process as in flight, with the exception of not recording acceleration data.

Table 1. Types of solder and flux tested.

\begin{tabular}{|c|c|}
\hline Solder and Flux Type & Manufacturer and Part Number \\
\hline Eutectic, No-Clean Flux Core & Kester \#245 No-Clean Flux Core \\
\hline Eutectic, Rosin Flux Core & Kester \#44 Rosin Core \\
\hline Solid Eutectic Solder with External, No-Clean Liquid Flux & Kester 959T Flux \\
\hline
\end{tabular}

\section{A. Data Analysis}

The analysis of the solder joint samples generated in both reduced and normal gravity requires a series of steps, outlined here. The first step focused on correlating the video and acceleration records of joint formation to select solder joints formed in reasonable acceleration environments. Joints passing these criteria were then inspected by a NASA technician for surface defects or indications of poor joint formation. These solder joints were then removed from the circuit board and sent for CT scanning, revealing the internal structure of the solder joint. Images from this analysis were then analyzed using a freely available software package for image analysis, with an analysis scheme developed by the authors.

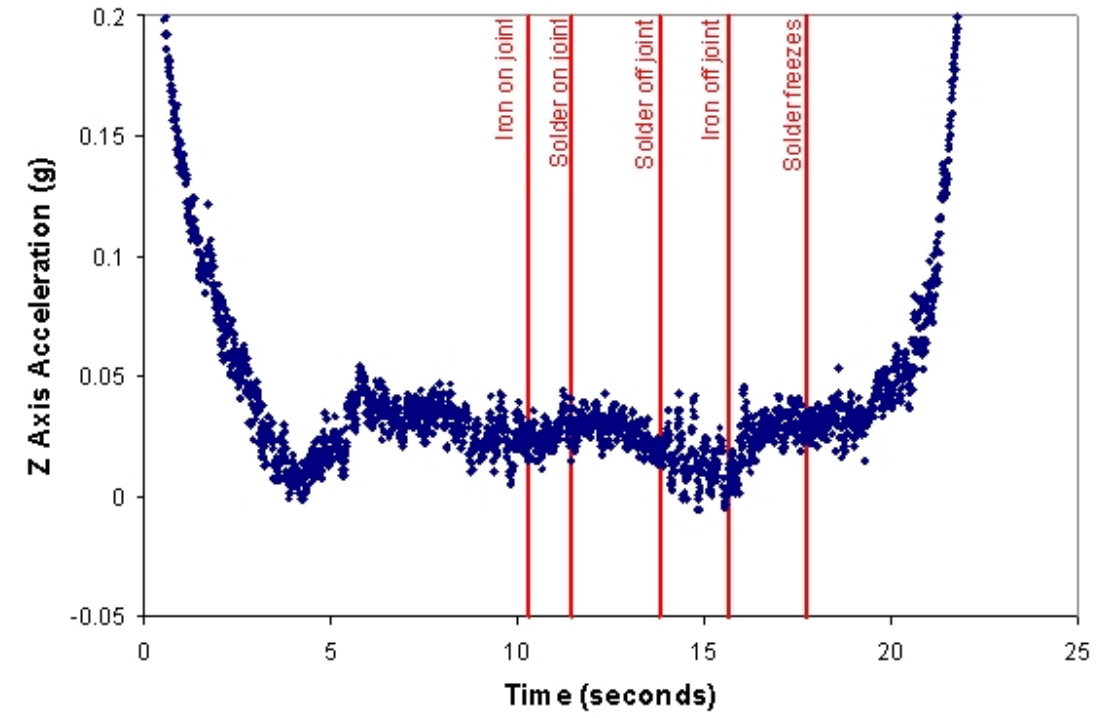

Figure 3. Graph of acceleration environment during a parabola on the NASA Reduced Gravity Research Aircraft.
Data analysis began with correlating the acceleration data with the recorded video data. Figure 2 shows a still image from the video record, providing an edge view of the solder joint during formation as well as the video overlays. This image also shows the three main features of a joint, the fillet that forms on the circuit board land and lead external to the circuit board, and the location of the annular region formed by the circuit board through hole and the component leg. As mentioned earlier, these video overlays allowed for correlating actions in the video record with the acceleration data. The goal is to select solder joints where gravitational level was both as

small as possible and as stead as possible while the solder was liquid. Figure 3 shows the acceleration data from one such joint, as well as indicators from the video record. These indicators show when the soldering iron is applied, when solder is applied (which marks the beginning of the critical time when molten solder is present), when the solder wire is removed, when the soldering iron is removed, and when the solder freezes, ending the critical joint formation period. This process removed many solder joints from consideration for analysis due to high overall gravity levels, "g-jitter" caused by turbulence, winds aloft, or flight profiles causing spikes in acceleration, or vibration within the aircraft negatively effecting the acceleration environment during the critical period when the solder is molten.

Next, a certified NASA technician inspected the solder joints that passed the acceleration criteria. The technician judged the joint quality, looking for surface defects, wetting defects, or flaws in the soldering operation. These criteria are based on standards established by NASA for solder joint formation and acceptability ${ }^{9}$. Solder joints passing both the acceleration and inspection criteria were sent for computed tomography (CT) scanning, revealing

$$
4
$$

American Institute of Aeronautics and Astronautics 
the internal structure of the solder joints. This process requires removing the solder joint from the circuit board, which is accomplished by cutting the component lead and using a small saw to cut a rectangular section of the circuit board containing the solder joint out of the circuit board.

The CT scans of solder joints are performed by Phoenix|X-Ray of St. Petersburg, Florida using their Nanotom instrument. The following is a brief description of the imaging process; a more detailed description can be found in the literature ${ }^{10,11}$. The sample is placed in a fixture between an $\mathrm{x}$-ray source and detector. The sample translates and rotates in a precise pattern, with x-ray images taken at each position. The image intensity at each position is a function of the density along the line of sight for the specific position. By combining these data, the technicians construct a three-dimensional model of the sample, including internal features. This model is composed of "voxels", or a cube similar to a pixel in standard two dimensional images. With the solder samples analyzed, the limiting voxel size is on the order of 10 micrometers per voxel side, with some variation from sample to sample due to sample preparation and mounting. Each three dimensional model allows for the generation of a series of two dimensional images, in this case an axial view (imaging along the component lead), a sagittal view (imaging perpendicular to the lead), and a second sagittal view normal to the first. This analysis used the axial view exclusively.

Figure 4 shows a sequence of images along the axial view from a solder joint. The first image shows the top of one fillet, with the dark lead in the center of the image surrounded by brighter solder. The next image shows a section of the same fillet, closer to the circuit board plane, demonstrating how the joint diameter increases along the fillet moving closer to the circuit board. The image also shows dark areas within the solder region. The experience of the CT company with similar work in the past indicates that these dark sections, distinguished from the component lead, are internal void defects. Future destructive testing will verify this
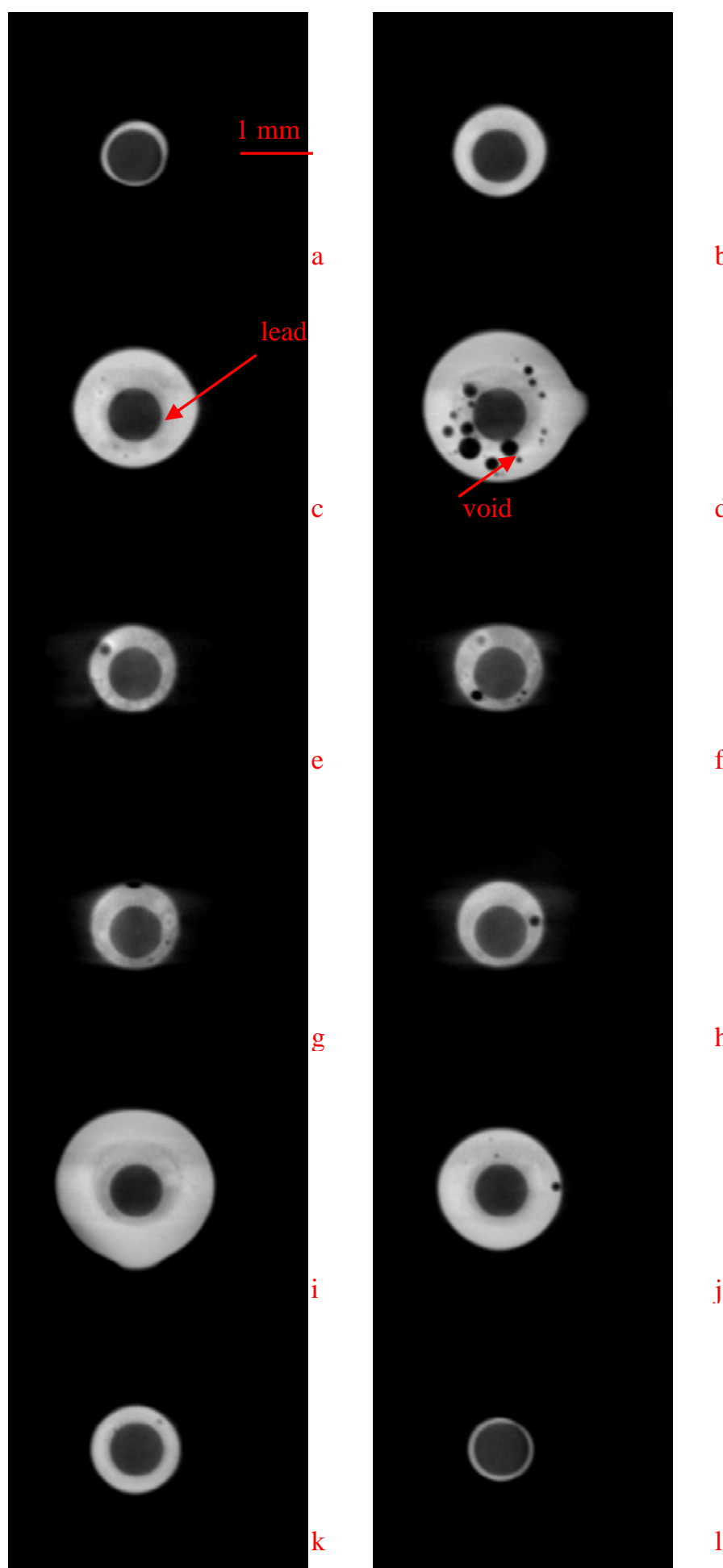

Figure 4. A sequence of images from solder joint 49-30, formed in reduced gravity using a no-clean flux cored solder. Images a-d are from one fillet with $0.25 \mathrm{~mm}$ between images, e$h$ are from the annular region with $0.43 \mathrm{~mm}$ between images, and $\mathrm{i}-\mathrm{I}$ are from the opposite fillet with $0.25 \mathrm{~mm}$ between images. The images also demonstrate the lead and an example of a void. 
hypothesis, but this data is not complete at this time. The next series of images show the solder joint structure within the circuit board through hole, or annular region. This clearly shows the lead, solder, and void defects, but the area of the joint is constrained by the circuit board. Finally, the next series of images shows the fillet on the opposite side of the circuit board, again with the largest solder diameter closest to the circuit board, with the area decreasing further from the circuit board. With the limits of x-ray instrument used to gather this data and the size of the solder

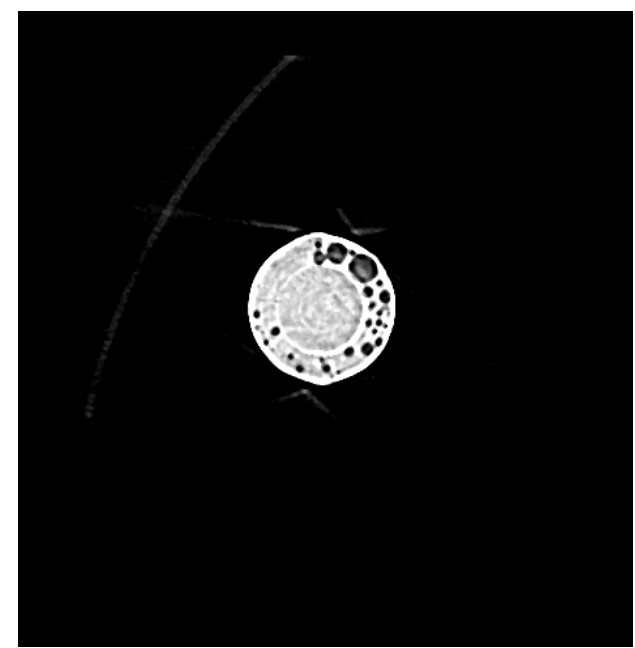

Figure 5. Solder joint image after performing "Unsharp Mask" and despeckle routines in ImageJ.

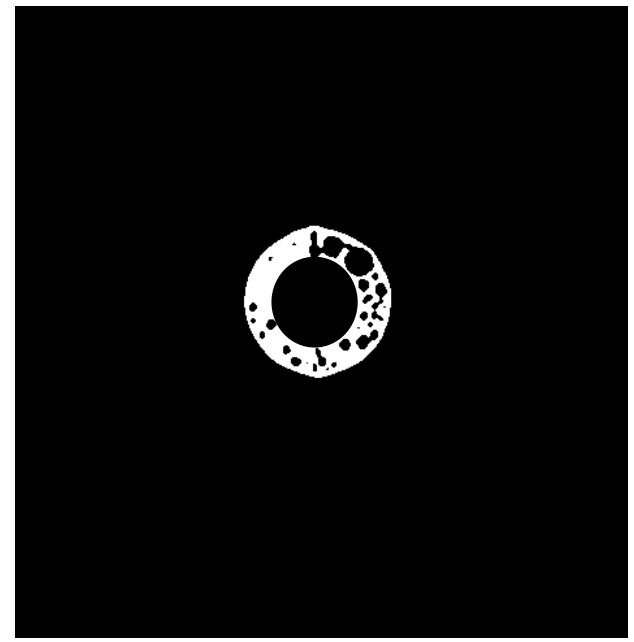

Figure 6. Solder joint image after thresholding to isolate voids. joints, a joint would typically require a sequence of 250-300 images to completely describe the solder joint.

Once the interior of the solder joint has been imaged, the next step is to analyze those images and determine the void fraction of the specific solder joint. The free software package Image ${ }^{12,13}$ provides a platform for analyzing the series of images generated by the CT analysis of a solder joint. The authors used the available macro language to create a script for analyzing a series of images. The first step in the analysis is to determine the total area of a solder joint. For the fillet regions, the algorithm used a threshold operation to isolate the bright solder region from the dark background, while counting the dark void regions enclosed in the solder. This is shown in Fig. 5. For the annular region, which has a constant area because it is constrained within the circuit board through hole, an oval tool bounding the joint area provided an area measurement. The next step was to measure the solder void area. The first step in this process used the "Unsharp Mask" feature of ImageJ to accentuate the intensity differences between dark objects (voids in this case) from bright objects, in this case the solder. Next, a despeckle operation replaces each pixel intensity with the median intensity of a 3-by-3 matrix centered on the pixel, eliminating noise introduced by the "Unsharp Mask" operation. After applying these two processes, a threshold operation separated voids from solder, and a watershed filter closed voids left open due to the void location near the joint or lead edge, shown in Fig. 6. This process reported a void plus lead area measurement for each slice of the joint. (The process could not distinguish between a void and the lead.) A final, manual measurement of the lead area, using an oval tool as in the task of measuring the joint area for the annular region, provided the last necessary information on the interior of a solder joint.

With these pieces of information - total joint area, void area, and lead area - an analysis of each slice and of the joint as a whole can begin. First, the lead area is subtracted from both the total area and the void area, as it is included in both measurements. The void fraction for each slice can then be calculated by dividing the void area by the total area, as shown in Eq. (1). Next, the total voiding is calculated as the sum from each image slice of the void areas divided by the sum of the total area, shown in Eq. (2). The average voiding is the mean of the void fraction across all slices in the solder joint. The data also allows for plotting the void fraction for each slice, indicating the locations within the solder joint with large amounts of void formation.

$$
\begin{array}{r}
\text { VoidFraction }=\frac{\text { VoidArea }}{\text { TotalArea }} \\
\text { TotalVoiding }=\frac{\sum_{\text {slices }} \text { VoidArea }}{\sum_{\text {slices }} \text { TotalArea }}
\end{array}
$$$$
6
$$ 


\section{Results}

The following section discusses the results from examining solder joints formed in both reduced and normal gravity eutectic solder wire and three flux combinations: no-clean flux cored wire, rosin flux cored wire, and solid wire with externally applied, liquid no-clean flux. All solder joints passed a visual workmanship inspection by a NASA certified electronics technician, exhibited low gravity levels and fluctuations (for joints formed in reduced gravity), and were internally analyzed using the techniques described earlier.
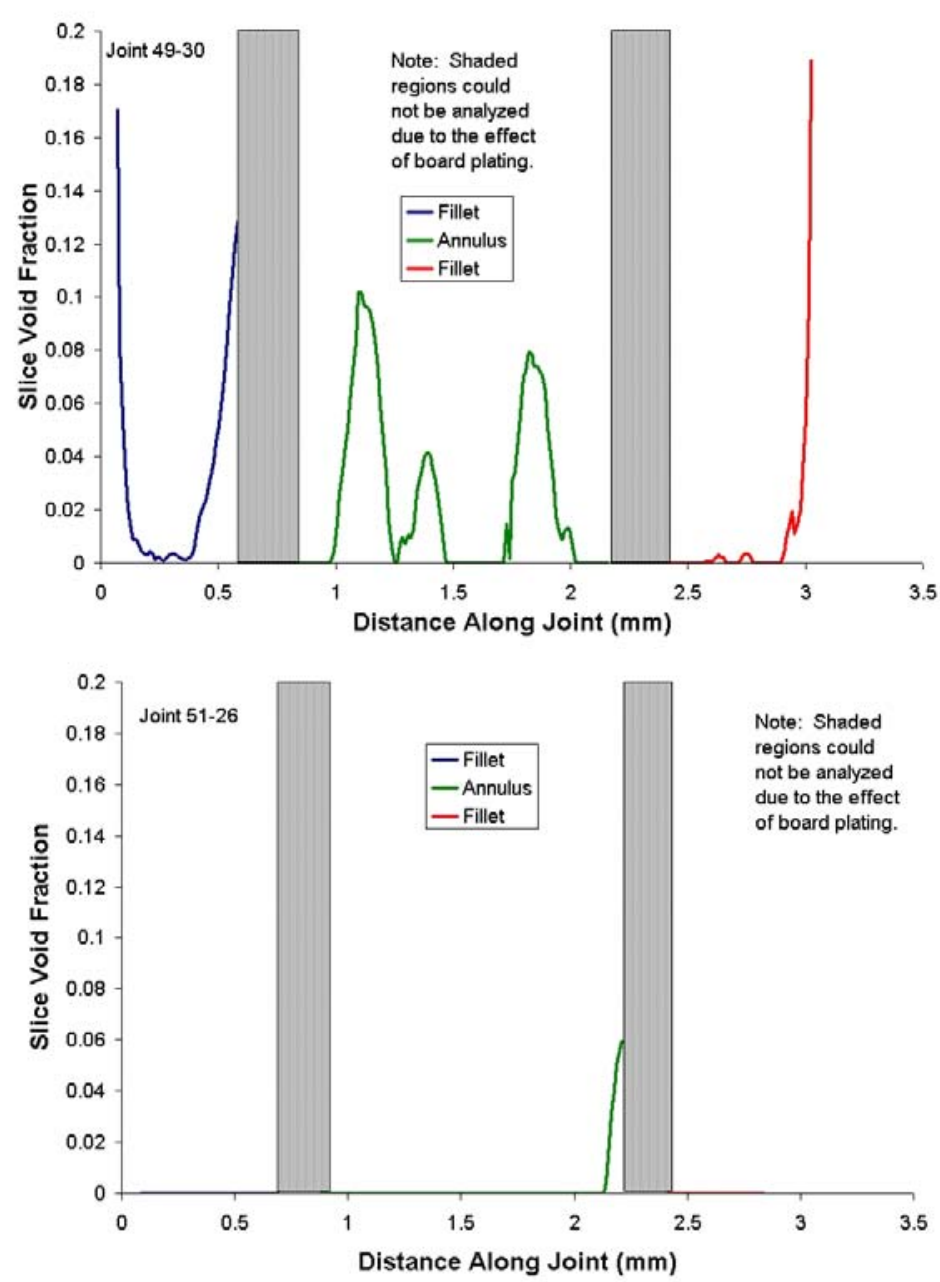

Figure 7. Void fraction for each slice of a no-clean flux core joint formed in reduced and normal gravity. Top figure is from reduced gravity, bottom from normal gravity.
Figure 7 shows the void fraction for each slice (given as distance along the joint) of two solder joints. Both solder joints used solder with a no-clean flux core, with one joint formed in reduced gravity (top) and the second formed in normal gravity (bottom). Each graph shows data from the three regions of the solder joint, namely each fillet and the annular throughhole region. The solder joints formed in reduced gravity showed void formation in both fillets and in the annular region, while joints formed in normal gravity showed most (but not all) voiding in the annular region. Void fraction at either end of the joint (small slice number or large slice number) can be large because the total solder area is small; voids forming in this area can represent unusually large void fractions because there is little solder in area. Voids may also form in a series of pockets, shown as the multiple peaks in the annular region of the reduced gravity joint. This is typical of all solder joints, and may occur in the fillets as well as the annular region. The plating on the circuit board did not seem to have an effect on the formation of voids in either reduced or normal gravity. The data show neither a consistent trend of void formation near the plated region, nor a consistent lack of void formation near the plating. The data in Fig. 7 support this, with some regions near circuit board plating showing void formation and some showing no void formation, in both reduced and normal gravity. Overall, joints formed in reduced gravity showed more overall void formation, and more regions of void formation, than joints formed in normal gravity. The data in Fig. 7 show much larger peaks in void fraction as well as more concentrations of void formation in the reduced gravity data set than the normal gravity set.

The data in Table 2 shows the average voiding, total voiding, and maximum slice void fraction for all joints analyzed that used eutectic solder wire with a no-clean flux core. Overall, solder joints made using this solder wire and flux combination form more voids when the joint forms in reduced gravity, compared to those formed in normal gravity. Average voiding for reduced gravity joints ranged from 1.5\% to $10.5 \%$, though 3 of the 4 joints presented had average voiding between $1.4 \%$ and $2.1 \%$. The total voiding for these reduced gravity joints ranged from $1.7 \%$ to $10.4 \%$, with most joints in the lower $1.7 \%$ to $3.2 \%$ region. Maximum slice void fraction ranged from $12.5 \%$ to $23.4 \%$, showing that for the worst slices one eighth to one quarter of the possible solder area was replaced with a void. Joints formed in normal gravity showed much smaller voiding characteristics. Both the average voiding and the total voiding in these cases was $0.1 \%$, with the largest maximum slice void fraction measured as $1.2 \%$. 
Table 2. Void fraction results for solder joints formed using eutectic solder with a no-clean flux core in reduced and normal gravity.

\begin{tabular}{|c|c|c|c|c|}
\hline $\begin{array}{c}\text { Joint } \\
\text { Number }\end{array}$ & $\begin{array}{l}\text { Gravity } \\
\text { Level }\end{array}$ & $\begin{array}{l}\text { Average Voiding } \\
(\mathbf{\%})\end{array}$ & $\begin{array}{l}\text { Total Voiding } \\
\mathbf{( \% )}\end{array}$ & $\begin{array}{l}\text { Maximum Slice Void Fraction } \\
\mathbf{( \% )}\end{array}$ \\
\hline $45-7$ & 0 & 1.8 & 1.7 & 13.4 \\
\hline $46-30$ & 0 & 10.6 & 10.4 & 23.4 \\
\hline $49-30$ & 0 & 2.1 & 2.1 & 18.9 \\
\hline $49-32$ & 0 & 1.4 & 3.2 & 6.5 \\
\hline $51-26$ & 1 & 0.1 & 0.1 & 1.2 \\
\hline $51-28$ & 1 & 0.1 & 0.1 & \\
\hline
\end{tabular}

The summary data presented in Table 3 presents the void formation data for solder joints formed using a rosin flux core. The average voiding in these cases ranged from $3.7 \%$ to $13.5 \%$, with 3 of the 4 joints analyzed exhibiting $6.9 \%$ average voiding or less. Total voiding ranged from $2.9 \%$ to $13.8 \%$, while the maximum slice void fraction ranged from $16.7 \%$ to $38.4 \%$. For the worst slices in the reduced gravity case, more than one third of the area available for solder was instead taken up by voids. The joints formed in normal gravity showed considerably less void formation with this flux than joints formed in reduced gravity. The average voiding was less than $0.5 \%$, total voiding less than $0.6 \%$, and the maximum slice void fraction was approximately $8.5 \%$ for these cases.

Table 3. Void fraction results for solder joints formed using eutectic solder with a rosin flux core in reduced and normal gravity.

\begin{tabular}{|c|c|c|c|c|}
\hline $\begin{array}{c}\text { Joint } \\
\text { Number }\end{array}$ & $\begin{array}{c}\text { Gravity } \\
\text { Level }\end{array}$ & $\begin{array}{c}\text { Average Voiding } \\
\mathbf{( \% )}\end{array}$ & $\begin{array}{c}\text { Total Voiding } \\
\mathbf{( \% )}\end{array}$ & $\begin{array}{c}\text { Maximum Slice Void Fraction } \\
\mathbf{( \% )}\end{array}$ \\
\hline $38-30$ & 0 & 3.7 & 2.9 & 16.7 \\
\hline $39-7$ & 0 & 6.5 & 7.2 & 38.4 \\
\hline $40-5$ & 0 & 13.5 & 13.8 & 36.1 \\
\hline $41-28$ & 0 & 6.9 & 9.2 & 24.4 \\
\hline $43-5$ & 1 & 0.2 & 0.2 & 8.4 \\
\hline $43-7$ & 1 & 0.5 & 0.6 & 8.6 \\
\hline
\end{tabular}

The data in Table 4 presents the average voiding, total voiding, and maximum slice void fraction for solder joints formed using a solid solder wire with an external, liquid no-clean flux. For the three reduced gravity cases analyzed the average voiding ranged from $6.2 \%$ to $38 \%$, with the total voiding ranging from $3.7 \%$ to $25.1 \%$. The maximum slice void fraction ranged from $23.7 \%$ to $84.1 \%$; in a region of the annulus for this joint (23-1), more than half of the area available for solder was instead taken by a void. The single normal gravity case analyzed here showed average voiding of $1 \%$, total voiding of $0.8 \%$, and a maximum slice void fraction of $15.1 \%$. These values are much smaller than those for reduced gravity cases using this solder and flux combination.

Table 4. Void fraction results for solder joints formed using eutectic solder with a no-clean external flux in reduced and normal gravity.

\begin{tabular}{|c|c|c|c|c|}
\hline $\begin{array}{c}\text { Joint } \\
\text { Number }\end{array}$ & $\begin{array}{c}\text { Gravity } \\
\text { Level }\end{array}$ & $\begin{array}{c}\text { Average Voiding } \\
\mathbf{( \% )}\end{array}$ & $\begin{array}{c}\text { Total Voiding } \\
\mathbf{( \% )}\end{array}$ & $\begin{array}{c}\text { Maximum Slice Void Fraction } \\
\text { (\%) }\end{array}$ \\
\hline $19-30$ & 0 & 11.1 & 13.3 & 32.1 \\
\hline $20-5$ & 0 & 6.2 & 3.7 & 23.7 \\
\hline $23-1$ & 0 & 38.0 & 25.1 & 84.1 \\
\hline $33-28$ & 1 & 1.0 & 0.8 & 15.1 \\
\hline
\end{tabular}

\section{Discussion}

The current results as well as previous research both show an increase in voiding for solder joints formed in reduced gravity compared to normal gravity. This previous work suggested a number of causes for an increase in void formation for solder joints created in reduced gravity compared to normal gravity ${ }^{6,7}$. One cause is the reduced effects of buoyancy in reduced gravity, increasing the amount of time required for a gas bubble generated during soldering to escape from the joint. In normal operations, the soldering iron is removed from the solder joint one second (or less) after the solder wire is removed. When this technique is used in reduced gravity, there is not enough time for gasses generated within the solder joint to escape before the solder solidifies, generating voids within the solder joint. In both the previous and current work the soldering iron remains on the solder joint for an extended 
period (approximately three seconds) after the solder wire is removed to provide more time for gas to escape the still-liquid solder joint. This increased heating time may have an adverse effect as it may allow continued volatilization of flux residue and vaporization of moisture trapped in the circuit card, which are potential sources of voids. Future work will be required to determine the optimum heating time.

Another difference between the previous work and the current work is the data analysis, specifically the previous analysis using a destructive technique where the solder joint is ground down to a specific plane (usually the joint midplane) and imaged ${ }^{6,7}$ as opposed to the current use of CT scanning, which images the entire volume of the joint without destroying the joint. The earlier technique required a large number of samples for statistical relevance, as each analyzed joint provided only one image of the joint interior (the previous work also required analysis of the acceleration environment and inspection for joint quality before analysis). The current analysis technique provides a much greater number of images per solder joint, providing more information on the joint structure. However, this work relies on a much smaller number of solder joints for analysis, which is desired due to limited availability of the NASA Reduced Gravity Research Aircraft. Future work must include a larger sample of solder joints for analysis using the techniques outlined here, to provide a larger sample population and greater confidence in the results.

These results could impact efforts currently underway to develop an electronics repair capability for future space missions $^{3-5}$. The selection of a solder and flux for use in these operations is an important part in allowing crew members to produce good, high reliability solder joints. The presence of voids within a solder joint can lessen the reliability of a solder joint, in terms of electrical connections, heat transfer, and mechanical attachment during high vibration or high forces. This must be balanced with the ease of use by an inexperienced crew person in an enclosed environment. While no-clean fluxes have, in this case, produced increased voiding, they are attractive for use because they do not require cleaning the circuit board and joint area after forming the solder joint. Rosin or other active fluxes are mildly acidic; over time, the residue from these fluxes can damage the solder joint, circuit board lands, or leads. Cleaning the circuit board requires the use of solvents, but this is problematic in an enclosed environment. Even relatively benign solvents, such as isopropyl alcohol, can damage other equipment and systems in a space craft, including air recycling and reclamation equipment. Future work must focus on reducing the presence of voids within solder joints using no-clean fluxes, but may also pursue a determination of the effects of voiding. This future work would focus on finding a potentially acceptable level of voiding, in terms of circuit reliability and robustness. If the amount of permissible voiding is small, other materials or techniques must be developed to reduce voids in solder joints produced in reduced gravity.

The small sample size in the current work makes identifying trends difficult in the data. The average voiding, total voiding, and maximum slice void fraction can vary widely from test to test. Future work should increase the number of solder joints of each type available for analysis, and use the analysis methods outlined in this paper. One flux type to focus on in this future work is the eutectic solder with a no-clean flux core. This solder type showed less void formation in both normal and reduced gravity than the other solder types. Characterizing a solder with no-clean flux would also aid in the overall work of developing a capability for performing electronics repairs during a long duration space mission, as these materials would not require cleaning, or reduced levels of cleaning, in the enclosed spacecraft, easing the burden on crew members, support equipment, and habitat.

\section{Acknowledgments}

The authors would like to thank Richard Pettegrew, formerly with the National Center for Space Exploration Research, Cleveland, $\mathrm{OH}$, and currently with IEC, Cleveland, $\mathrm{OH}$, for specific assistance in performing these experiments as well as many years of effort on this project. The authors would like to acknowledge the efforts of the Reduced Gravity Aircraft Facility at NASA Johnson Space Center, Houston, TX, for their help in readying the experiment rig for operation and providing a platform for performing the experiments. Thanks also go to Gary Gorecki, NASA Glenn Research Center, Cleveland, $\mathrm{OH}$, for his efforts in preparing the circuit board and solder wire samples, as well as preparing the aircraft rig for operation. This work is performed as a part of the Component Level Electronic Assembly Repair (CLEAR) project at NASA Glenn Research Center, Cleveland, OH, managed by Duc Truong. Funding for this work is through the Exploration Technology and Development Program, managed by Barmac Talaghani at NASA Langley Research Center, Langley, VA.

\section{References}

${ }^{1}$ Oberg, James, “Space Station: Internal NASA Reports Explain Origins of June Computer Crisis”, IEEE Spectrum, October 4, 2007. 
2 Spotts, Peter N., “A Reality Check On Dreams For Space: The Repairs”, Christian Science Monitor, November 5, 2007.

${ }^{3}$ Pettegrew, R.D., Easton, J.W., Struk, P.M., Anderson, E.E., “In-Flight Manual Electronics Repair for DeepSpace Missions”, 2007 IEEE Aerospace Conference, March 3-10, 2007, Big Sky, MT, Paper\#1208 (2007).

${ }^{4}$ Pettegrew, R.D., Easton, J.W., and Struk, P.M., "Repair of Electronics for Long Duration Spaceflight”, $45^{\text {th }}$ AIAA Aerospace Science Meeting and Exhibit, Reno, NV, January 8-11, 2007, AIAA-2007-1364 (2007).

${ }^{5}$ Easton, J.W., Pettegrew, R.D., and Struk, P.M., “Electronic Repair Concepts for Long-Duration Spaceflight”, $4^{\text {th }}$ AIAA Aerospace Sciences Meeting and Exhibit, Reno, NV, January 8-11, 2007, AIAA-2007-545 (2007).

${ }^{6}$ Watson, J.K., Struk, P.M., Pettegrew, R.D., and Downs, R.S., Experimental Investigation of Solder Joint Defect Formation and Mitigation in Reduced-Gravity Environments, AIAA Journal of Spacecraft and Rockets, Vol. 44, No. 1, pp. 174-182, (Jan-Feb 2007).

${ }^{7}$ Pettegrew, R.D., Struk, P.M., Watson, J.K., Haylett, D.R., and Downs, R.S. Gravitational Effects on Solder Joints, Welding Journal, pp. 44-48 (2003).

${ }^{8}$ JSC Reduced Gravity Program User's Guide, AOD 33899, August, 2005.

${ }^{9}$ Soldered Electrical Connections, NASA-STD-8739.3, 1997.

${ }^{10}$ Kak, Avinash C., and Slaney, Malcolm, Principles of Computerized Tomographic Imagining, IEEE Press, 1988.

${ }^{11}$ Brunke, O., Odenbach, S., Jurgons, R., Alexiou, C., Hilger, I., and Beckmann, F. J. Phys.: Condes. Matter, Vol 18, S2903-S2917, 2006.

12 Rasband, W.S., ImageJ, U. S. National Institutes of Health, Bethesda, Maryland, USA, http://rsb.info.nih.gov/ij/, 1997-2007.

13 Abramoff, M.D., Magelhaes, P.J., Ram, S.J. "Image Processing with ImageJ". Biophotonics International, volume 11, issue 7, pp. 36-42, 2004. 